\title{
Research on Fishery Water Quality Detection System of Unmanned Aerial Vehicle
}

\author{
Han $\mathrm{Cao}^{1}$, Lei Cheng1*, Xin Wang ${ }^{1}$ and Quanmin $\mathrm{Zhu}^{2}$ \\ ${ }^{1}$ Wuhan University of Science and Technology, School of Information Science and Engineering, China \\ ${ }^{2}$ Department of Engineering Design and Mathematics, University of the West of England, Bristol; BS16 1QY, UK \\ *Corresponding author: Lei Cheng, Wuhan University of Science and Technology, School of Information Science and Engineering, \\ Wuhan 430081, P.R.China
}

\begin{abstract}
Many lands in China are close to rivers and oceans and they are very suitable for developing fisheries. Water quality monitoring is indispensable to the development of fisheries. Therefore, a method of detecting, evaluating and predicting fishery water quality using unmanned aerial vehicle electrode sensor array is proposed. The results show that this method can timely monitor fishery water quality and predict the future trend of water quality and improve the detection efficiency.
\end{abstract}

Keywords: Fishery water; Prediction; Electrode Sensor Array; UAV

\section{Introduction}

Among the standards for measuring fishery water quality [1], the PH value of fishery water, ammonia nitrogen content, temperature, dissolved oxygen are important data indicators for traditional fish farming, which have a direct impact on fish farming. In the traditional analysis and prediction of fishery water quality, it takes a long time to sample fishery water and then carry out physical and chemical analysis, which will greatly delay the timely management of fishery water and resulting in irreversible losses. Therefore, it is necessary to find a faster, more convenient and reliable method. As far as we know, the electrode electrochemical sensors have the characteristics of fast equivalent circuit network, high selectivity of measurement, the excellent waterproof performance and corrosion resistance. It is an excellent choice for fishery water quality monitoring. At the same time, due to UAV's [2] high mobility and flexibility, outstanding performance and practical application in the agricultural field, UAV has expanded our thinking of using it in the fishery field. In this paper, we focus on the application of electrodetype chemical water quality sensors in fishery production practice [3], and carry them on the platform, and transmit the data to the cloud database through GPRS network for LSTM [4], in order to achieve more accurate and rapid assessment and prediction of fishery water quality in modern aquaculture.

\section{Methods}

\section{UAV Suitable for Water Surface}

In order to ensure UAV to float smoothly on the water surface and leave the water quickly, the floating structure can be made of ethylene-vinyl acetate copolymer (EVA). EVA [5] is a polymeric material whose molecular formula is $(\mathrm{C} 2 \mathrm{H} 4) \mathrm{x}$. (C4H6O2) $\mathrm{y}$, which is made of foam after foaming. It has the characteristics of closed cell structure, no water absorption, good water resistance, corrosion resistance, high resilience and high tensile resistance and EVA material has a good processing property and is easy to process floating structure.

\section{Electrode Chemical Sensor Array}

Most of the electrode chemical sensors are based on chemical electrolyte as the medium, belonging to the primary battery system. After contacting fishery water, the substances to be measured are exchanged through the thin film, and the electrolyte inside can 
take place electrolytic reaction to produce corresponding electrical signals. For different detection components, the electrolyte inside the electrode chemical sensor is different, and the film material is selective, so the electrode chemical sensor has the characteristics of high selectivity and reliability. Although the temperature will affect the sampling results of multiple electrode chemical sensors. However, by integrating the electrode chemical sensor with the temperature sensor, the data measured by the electrode chemical sensor can be corrected by the temperature sensor.

\section{Collection of Fishery Water Data}

We use remote controllers, computer terminal ground stations and GPS satellite positioning to allow UAV to fly on planned routes and land accurately in the water quality area to be measured. After the UAV is parked on the water surface, sensors hidden in EVA materials begin to monitor the water quality. Each group of data contains temperature and content. There are three groups of data. After average processing, the data are transmitted to the cloud server database, which is aggregated into the server database at intervals. During floating sampling, data is transmitted only to the cloud server. After floating sampling, the collected data are sent back to the back end, and the data are evaluated and processed. The final results will be displayed at the corresponding ports of the ground station. The original working data obtained in an evaluation operation are shown in the following Figure 1.

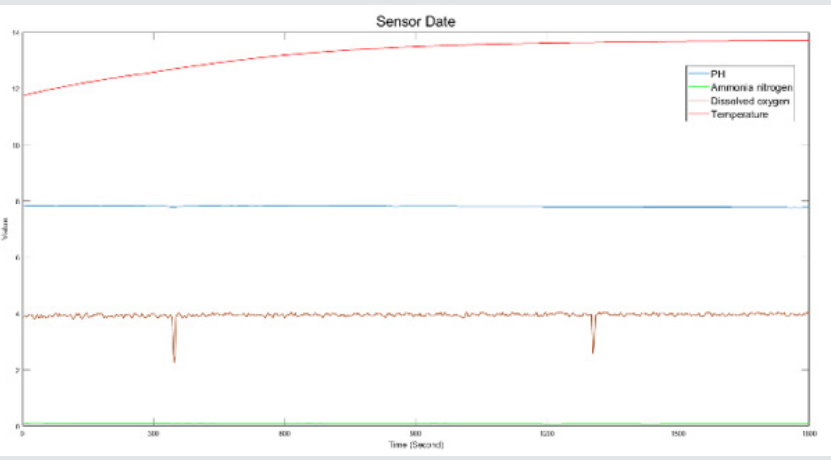

Figure 1: The original data measured by the sensor.

\section{Data Processing and Results}

The four indicators measured by the sensor are uploaded to the cloud server after taking the average value, and the data are uploaded every 3 seconds. After every period of time, the data uploaded during that period are aggregated at the back end, and the aggregated data are processed. The processed experimental data are compared with the fishery water quality measurement standard, and the possible results are predicted, and the phase is given. The corresponding solution. Data processing mainly uses LSTM algorithm. LSTM can perform better in a longer sequence than ordinary RNN. LSTM differs from RNN in that it adds a "processor" to the algorithm to judge whether information is useful or not. The structure of the processor is called cell. According to the fishery water quality measurement standard, we divide the water quality grade into the following tables: (Table 1). Through the analysis of the original working data, it can be concluded that $\mathrm{PH}$ is basically stable between 7.7 and 7.9, DO is about 3.8-3.9, $\mathrm{NO}_{2}$ is between $0.09-0.1, \mathrm{NH}_{4}+$ is between 0.1 and 0.11 , the temperature is increasing, and the fishery water level is 2 . The experimental results after treatment are extracted as follows: (Table 2). It can be seen from the table that the experimental results after treatment are basically consistent with the analysis.

Table 1: Classification.

\begin{tabular}{|c|c|c|c|c|}
\hline & $\mathbf{P H}$ & $\mathbf{D O}$ & $\mathbf{N O}_{\mathbf{2}^{-}}$ & $\mathbf{N H}_{4}{ }^{+}$ \\
\hline $1(100)$ & $6.5-8.5$ & $5-8$ & $<0.1$ & $<0.2$ \\
\hline $2(80-100)$ & $\begin{array}{c}5.5-6.5 \text { or } \\
8.5-9.5\end{array}$ & $3.5-5$ or $>8$ & $0.1-0.5$ & $0.2-0.5$ \\
\hline $3(<80)$ & $<5.5$ or $>9.5$ & $<3.5$ & $>0.5$ & $>0.5$ \\
\hline $\begin{array}{c}\text { weight } \\
\text { coefficient }\end{array}$ & 0.3 & 0.3 & 0.2 & 0.2 \\
\hline
\end{tabular}

Table 2: Analysis result.

\begin{tabular}{|c|c|c|c|c|}
\hline & PH & Do & $\mathbf{N O}_{2}{ }^{-}$ & $\mathbf{N H}_{4}{ }^{+}$ \\
\hline $2(87.8)$ & 7.83 & 3.86 & 0.09 & 0.1 \\
\hline
\end{tabular}

\section{Summary}

This paper reviews the composition of UAV water quality monitoring system and its corresponding algorithm, analyses in detail the details of UAV water quality monitoring system operation and introduces the application of LSTM algorithm in it. From the current performance effect, the UAV water quality monitoring system has a significant effect, which also confirms the shortcomings of the traditional fishery water quality monitoring system.

\section{References}

1. Shihong X, Xinrong W, Shitao X (2005) The role of fishery water quality analysis and monitoring in aquaculture. Jiangxi Aquatic Science and Technology 2005 (4): 6-9.

2. Vachtsevanos G J, Tang L, Drozeski G, Luis Gutierrez (2005) From mission planning to flight control of unmanned aerial vehicles: Strategies and implementation tools. Annual Reviews in Control, 2005, 29(1): 101-115.

3. Nuñez L, Cetó X, Pividori MI (2013) Development and application of an electronic tongue for detection and monitoring of nitrate, nitrite and ammonium levels in waters. Microchemical Journal 110(9): 273-279.

4. Liang YG, Song Z W, Tui, D (1988) The correlation between properties and structure of ethylene-vinyl-acetate (EVA) copolymers. Proceedings of the $21^{\text {st }}$ Symposium on Electrical Insulating Materials, Japan, pp. 5963.

5. Greff K, Srivastava RK, Koutník J, Bas R Steunebrink, Jürgen Schmidhuber (2015) LSTM: A Search Space Odyssey. IEEE Transactions on Neural Networks \& Learning Systems 28(10): 2222-2232. 


\begin{tabular}{l} 
Advances in Robotics \& \\
Mechanical Engineering \\
Assets of Publishing with us \\
- Global archiving of articles \\
\hline Robotics \& Mechenical Engineering
\end{tabular}

DOI: 10.32474 /ARME.2019.01.000122 